\title{
Posizionamento di un catetere venoso centrale permanente nella trombosi della vena cava superiore
}

\author{
Marina Cornacchiari ${ }^{1}$, Roberto Ferraresi ${ }^{2}$, Barbara Gidaro ${ }^{3}$, Antonia Stasi ${ }^{1}$, \\ Maria Giuseppina Ponticelli ${ }^{1}$, Marco Mereghetti ${ }^{1}$, Luca di Toma ${ }^{3}$, Carlo Maria Guastoni ${ }^{3}$
}

${ }^{1}$ Reparto di Nefrologia e Dialisi, Azienda Ospedaliera Legnano, Presidio Magenta, Legnano (MI)

${ }^{2}$ Laboratorio di Emodinamica Interventistica Periferica, Humanitas Gavazzeni, Bergamo

${ }^{3}$ Reparto di Nefrologia e Dialisi, Azienda Ospedaliera Legnano, Legnano (MI)

\begin{abstract}
Placement of permanent Central venous Catheters in thrombosis of the Superior Vena CaVa
Abstract. In the last years, the placement of permanent central venous catheters for hemodialysis, infusion, and electrophysiological therapy has widely increased in clinical practice. As a result, the number of central venous obstructions has increased due to thrombosis or reactive stenosis correlated with these devices.

This article describes the clinical case of an extensive thrombosis involving the superior vena cava and the anonymous trunks in a patient reliant on a permanent central venous catheter for hemodialysis treatment.
\end{abstract}

Key words: Thrombosis, Superior vena cava, Central venous permanent catheter, Hemodialysis

Conflict of interest: None.

Financial support: None.

Accettato: 28 Gennaio 2014

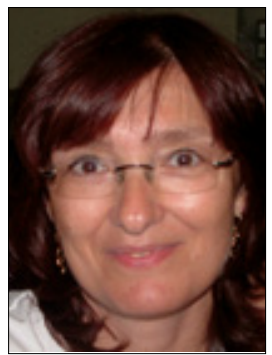

Marina Cornacchiari

\section{Introduzione}

È sempre più frequente, nella pratica clinica, il posizionamento, nei grossi vasi venosi centrali, di cateteri che sono utilizzati per l'emodialisi, la terapia infusionale, la nutrizione parenterale, la chemioterapia o la terapia elettrofisiologica delle aritmie e dello scompenso cardiaco. La conseguenza è la possibilità di provocare l'occlusione delle vene dove tali dispositivi alloggiano. La trombosi può anche rimanere clinicamente silente ed evidenziarsi solo in occasione di esami angiografici, eseguiti per motivazioni diverse. In questo articolo, si descrivono il caso clinico di una trombosi estesa, coinvolgente la vena cava superiore e i tronchi anonimi, in una paziente in cui l'impianto di un catetere venoso centrale permanente $(\mathrm{CVCp})$ era l'unica possibilità di proseguire il trattamento dialitico sostitutivo, e le modalità con cui il posizionamento è stato attuato.

\section{Caso clinico}

Donna di 63 anni, affetta da diabete mellito tipo II e retinopatia secondaria, ipertensione arteriosa e arteriopatia obliterante agli arti inferiori IV stadio. La comparsa di insufficienza renale cronica (IRC) secondaria a nefropatia diabetica e nefroangiosclerosi ha richiesto l'inizio delle terapia sostitutiva con dialisi peritoneale che si complicava nel tempo, con numerosi episodi peritonitici.

Nel Maggio del 2012, a seguito di un ennesimo grave episodio infettivo, si rende necessaria una sospensione in tempi brevi della dialisi peritoneale con passaggio all'emodialisi. La possibilità di poter riprendere in un successivo futuro la metodica peritoneale è negata dal fatto che la paziente è divenuta ipovedente e che $\mathrm{i}$ parenti non possono assisterla nell'effettuare la metodica.

Nel nostro reparto, il posizionamento dei CVC è eseguito in una saletta preposta a tale scopo sotto guida ecografica, ma senza la possibilità contestuale di un controllo fluoroscopico. Nel caso in oggetto, la sede del posizionamento è valutata con ecocolordoppler, che mostra una trombosi della vena giugu- 


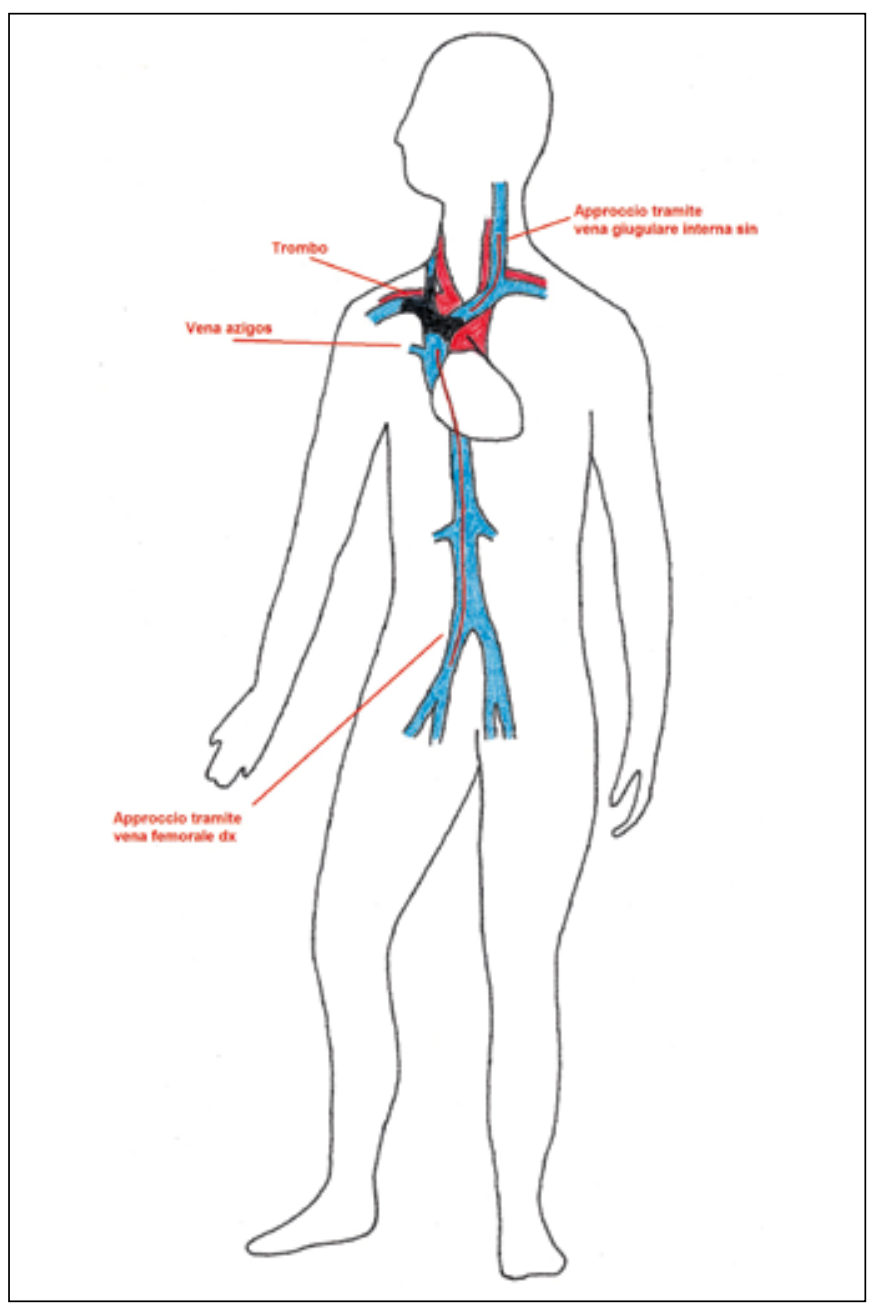

Fig. 1

lare interna e succlavia destra e la pervietà ecografica dei vasi controlaterali.

A tale livello, la curva velocimetrica mostra un flusso venoso discretamente modulato dal respiro, come segno indiretto di pervietà a valle, e, all'esame obiettivo, non sono presenti circoli venosi collaterali particolarmente evidenti, sulla parete toracica, tali da indurre il sospetto di una problematica di tipo ostruttivo a livello della vena cava superiore.

Si procede quindi, in urgenza, a posizionare un CVC temporaneo per la dialisi. Il tentativo del posizionamento del CVC nella giugulare interna sinistra, però, fallisce per la mancata progressione della guida metallica oltre i primi $10 \mathrm{~cm}$ dal punto di venipuntura. Questo pone il sospetto di un'occlusione dei vasi venosi centrali, che è confermato dall'angio TAC, che mostra una trombosi dei tronchi anonimi e della vena cava superiore fino alla confluenza dell'azygos.

La paziente è inviata in urgenza dal cardiologo interventista, con cui collaboriamo da anni, che, dopo venipuntura della giugulare interna sinistra, procede a introdurre una guida metallica 0.035 ". Attraverso la vena femorale destra, è introdotta un'altra guida metallica 0.035 ", che viene fatta risalire attraverso le cavità

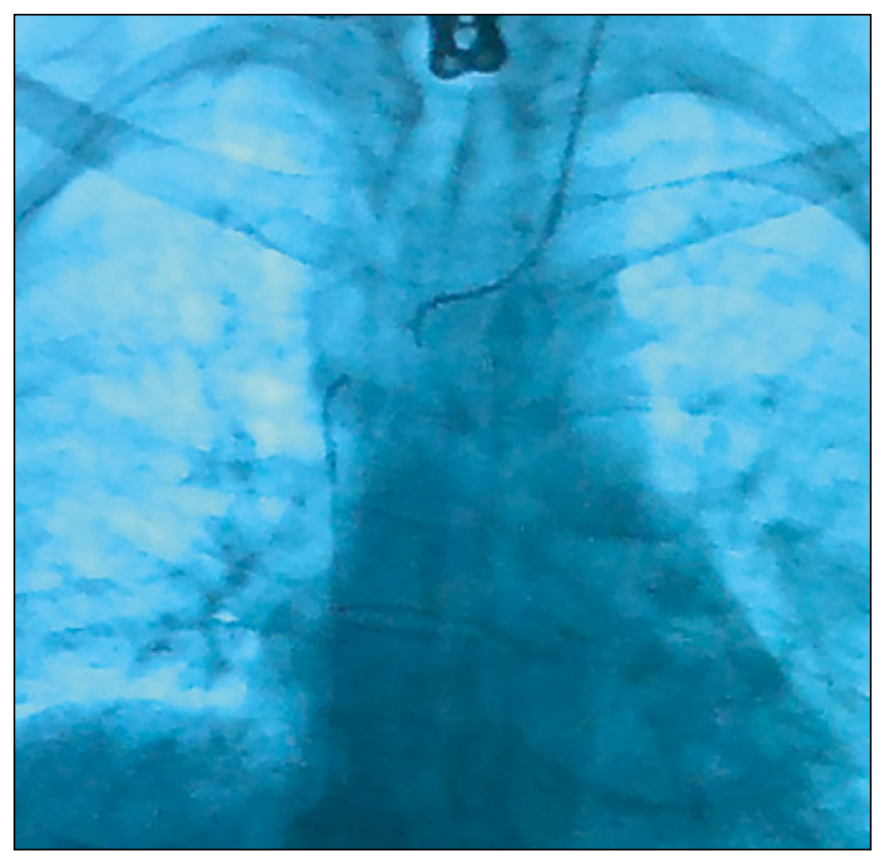

Fig. 2

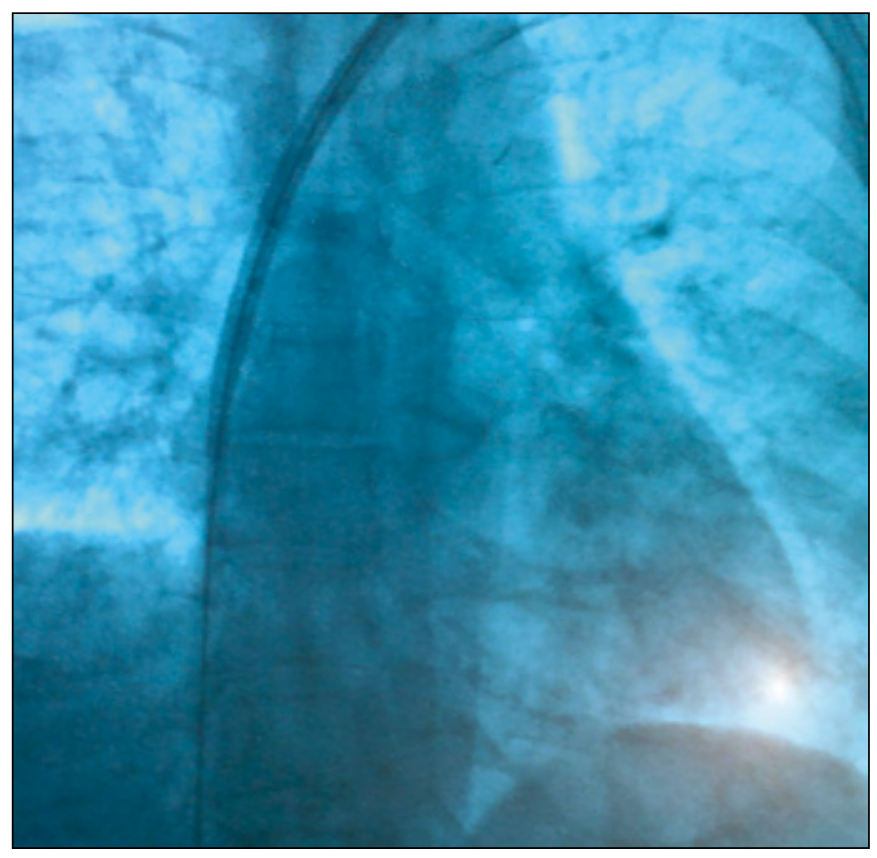

Fig. 3

cardiache, fino alla vena cava superiore (Figg. 1,2). Sulle due guide vengono fatti avanzare introduttori di lunghezza adeguata che vengono posizionati l'uno nel tronco anonimo sinistro, subito a monte dell'occlusione completa, e l'altro nella vena cava superiore, subito a valle della stessa. Un'angiografia ottenuta mediante doppia iniezione di mezzo di contrasto iodato evidenzia chiaramente l'anatomia: entrambi i tronchi 
anonimi sono occlusi e la vena cava superiore riceve il flusso ematico attraverso la vena azygos e la distanza tra il lume pervio del tronco anonimo sinistro e la vena cava superiore è di circa $1 \mathrm{~cm}$. Avanzando attraverso gli introduttori guide 0.018 " supportate da cateteri diagnostici di tipo Berenstein 4 $\mathrm{Fr}$, si cerca di avvicinare i due approcci, riuscendo, infine, a superare delicatamente l'occlusione. Si predilata il tratto occluso con un pallone sottodimensionato (diametro $4 \mathrm{~mm}$ ) per verificare la bontà del tragitto effettuato e si osserva la ripresa del flusso anterogrado in assenza di stravasi extra-vascolari di mezzo di contrasto. Si procede, pertanto, alla dilatazione del tratto stenotico con un pallone di diametro di $8 \mathrm{~mm}$ a $8 \mathrm{~atm}$. Attraverso l'introduttore superiore viene posizionato il CVCp, bilume, con l'apice nell'atrio destro (Fig. 3).

La paziente è rimandata al reparto di degenza. La comparsa di un ematoma a livello di parete toracica-spalla sinistra, conseguente alla manovra, ci induce a non iniziare la terapia con eparina a BPM a basso dosaggio, consigliata dal cardiologo interventista. A distanza di 48 ore, compare un edema a mantellina e un nuovo esame angio TAC mostra la riocclusione del tronco anonimo sinistro (intorno al CVCp) e la trombosi del tratto prossimale della vena azygos. Il CVCp, in precedenza ben funzionante, risulta trombizzato. È iniziata la terapia con eparina a BPM, a dosaggio scoagulante.

La paziente è nuovamente inviata dal cardiologo interventista, che procede a introdurre una guida metallica 0.035 " attraverso il CVCp che è, poi, rimosso. Sulla via della guida metallica sono inseriti un introduttore $10 \mathrm{Fr}$ e, quindi, uno stent auto espandibile, in nitinolo, con diametro $16 \mathrm{~mm}$, che viene posizionato a cavaliera di vena cava superiore e tronco anonimo sinistro. Si post-dilata con pallone $14 \mathrm{~mm}$ a $8 \mathrm{~atm}$, ottenendo un'ottima espansione dello stent. Infine, attraverso lo stent, si riposiziona un CVCp bilume per emodialisi con apice nell'atrio destro.

L'angiografia mostra la ricanalizzazione dell'azygos al controllo eseguito durante la procedura.

L'edema a mantellina si risolve progressivamente e la paziente, a tutt'oggi, esegue sedute emodialitiche efficienti con il CVC.

\section{Discussione}

L'utilizzo dei CVC è in costante aumento in tutte le nefrologie per molteplici ragioni (1). È in costante aumento anche per i pazienti non nefrologici, che hanno necessità, durante il ricovero, di posizionare degli accessi venosi centrali per problematiche varie (terapie infusive e parenterali, chemioterapie, ecc.). Numerosi, poi, sono i pazienti portatori di pacemaker e/o defibrillatori.

Lo sviluppo di trombosi dei vasi venosi centrali non è, quindi, un evento raro e, verosimilmente, è sottostimato (2), in quanto la trombosi può presentarsi anche come clinicamente silente. Nei pazienti nefropatici, la presenza di tali trombosi più o meno estese può costituire una grossa problematica quando il posizionamento di un CVCp è l'unica via percorribile per poter effettuare un trattamento depurativo, come nel caso clinico riportato sopra. Numerose sono le strade che si cerca di percorrere per poter trattare il paziente, dal posizionamento di CVC transepatici o nella vena cava inferiore (3) al posizionamento di stent attraverso i quali è inserito il CVCp (4).
$\mathrm{Nel}$ caso clinico riportato sopra, una trombosi che interessa la confluenza dei vasi anonimi e il tratto superiore della vena cava viene superata con l'impiego di uno stent e con il successivo posizionamento in esso di un CVCp.

Il caso descritto sopra, la metodica utilizzata dal radiologo interventista (nel nostro caso, un cardiologo interventista) e i risultati ottenuti ci spingono alle considerazioni riportate qui di seguito. La particolarità del caso è data dalla metodica utilizzata che possiamo riassumere in alcuni punti chiave:

1. Procedure di questo tipo devono essere effettuate in centri (ad alto volume di casi) esperti in questa tematica e attrezzati con grandi angiografi e che hanno a disposizione tutta la varietà di materiali necessari per affrontare e risolvere eventuali complicanze come la perforazione dei vasi trattati;

2. Lo studio angiografico dettagliato è l'elemento essenziale per guidare la strategia di ricanalizzazione. La ricerca di segni radiologici come difetti di riempimento (i cosiddetti "tram-track") è fondamentale per orientare le manovre volte al superamento dell'occlusione;

3. La dilatazione delle vene centrali si associa quasi sempre a un dolore toracico, pertanto deve essere eseguita in modo progressivo solo in presenza della documentata certezza di essere nel lume vero e con la possibilità di dominare, se necessario, il dolore. Lo stenting delle vene centrali pone numerose problematiche, sia per l'assenza di stent specifici per questo particolare distretto che per possibili problemi successivi (5).

Riteniamo corretto evidenziare anche alcuni inconvenienti (con le relative cause) occorsi durante la procedura. La comparsa di un edema a mantellina per l'occlusione dell'azygos attraverso cui, in precedenza, drenava la gran parte del sangue refluo da capo e arti è stata la conseguenza di due fattori: in primo luogo, la procedura è stata effettuata con la sola angioplastica con pallone sottodimensionato, pertanto il flusso post-procedurale attraverso l'anonima era modesto e, in secondo luogo, la terapia scoagulante consigliata non è stata iniziata immediatamente, ma è stata posticipata di oltre 48 ore. Questo ha determinato la trombosi del CVCp e, soprattutto, dell'origine dell'azygos. Fortunatamente, tale trombosi si è rapidamente risolta con l'uso di adeguati dosaggi di eparine a BPM. La funzionalità del CVCp è stata ristabilita con la sua sostituzione, in concomitanza con la nuova dilatazione, con un pallone di diametro finalmente corretto e con il posizionamento dello stent.

\section{Conclusioni}

Il caso clinico riportato sopra vuole sottolineare la necessità, per il nefrologo che si occupa in prima persona degli accessi vascolari, di individuare un angioradiologo (interventista, emodinamista) con il quale dialogare e interagire per affrontare alcune problematiche complesse legate al posizionamento o alla rimozione di CVCp. Procedure come quella descritta, per niente banali, richiedono una sala angiografica opportunamente attrezzata e colleghi specialisti esperti che, condividendo con il nefrologo la coscienza dell'importanza che riveste l'accesso vascolare per il paziente dializzato, non lesinino la propria disponibilità e il proprio impegno a risolvere $i$ vari casi. 


\section{Riassunto}

È sempre più frequente l'utilizzo di cateteri posizionati nei vasi venosi centrali per il trattamento emodialitico, la terapia infusiva, la nutrizione parenterale e la chemioterapia. Anche i cardiologi ricorrono sempre più spesso all'impianto di cateteri venosi centrali per l'elettrostimolazione e la cardioversione elettrica. Il risultato è la possibilità di comparsa dell'occlusione di tali vene a causa della presenza di questi dispositivi. Questo articolo descrive il caso clinico di un'ampia trombosi coinvolgente la vena cava superiore e la modalità di posizionamento di un catetere venoso centrale, unica possibilità per poter effettuare un trattamento dialitico nel paziente.

Parole chiave: Trombosi, Vena cava superiore, Catetere venoso centrale permanente, Emodialisi
Dichiarazione di conflitto di interessi: Gli Autori dichiarano di non avere conflitto di interesse.

Contributi economici agli Autori: Gli Autori dichiarano di non aver ricevuto sponsorizzazioni economiche per la preparazione dell'articolo.

Indirizzo degli Autori:

Dr.ssa Marina Cornacchiari

Servizio di Nefrologia e Dialisi

Ospedale G. Fornaroli

Via Donatore di Sangue 51

20013 Magenta (MI)

lisalo@tiscalinet.it

\section{Bibliografia}

1. Chaudhry M, Bhola C, Lok CE, et al. Seeing eye to eye: the key to reducing catheter use. J Vasc Access 2011; 12 (2): 120-6.

2. Gonsalves CF, Eschelman DJ, Bonn J, et al. Incidence of Central Vein Stenosis and Occlusion Following Upper Extremity PICC and Port Placement. Cardiovasc Intervent Radiol 2003; 26: 123-7.
3. Sewell PE Jr, Lawson ED, Tanner JD. Placement of Transmediastinal Dialysis Catheters Using MR Imaging Guidance. AJR 2001; 176 (1): 233-4.

4. Lorenz JM. Use of Stents for the Maintenance of Hemodialysis Access. Semin Intervent Radiol 2004; 21 (2): 135-40.

5. Kundu S. Treating CVD in the Dialysis Patient. Endovascular Today 2010; 60-5.

\section{TEST DI VERIFICA}

1) La trombosi completa della vena cava superiore può decorrere in modo completamente asintomatico?
a) Sì
b) $\mathrm{No}$ dei vasi venosi centrali?
a) Impianto di pacemaker
b) Cateteri infusionali
c) Cateteri per dialisi
d) Tutti
e) Nessuno

2) Quali procedure possono essere responsabili di trombosi
3) Nel caso clinico, il flusso venoso di capo e arti superiori era drenato tramite:

a) Vena azygos

b) Vena toracica interna
Le risposte corrette alle domande sono pubblicate su questo numero del Giornale di Tecniche Nefrologiche \& Dialitiche Vol. 26, no. 1, pag. 41. 